\title{
Platelet Distribution Width is Associated with Type 2 Diabetes Mellitus and Diabetic Nephropathy and Neuropathy
}

\author{
Burcin M. Atak*, Tuba T. Duman, Gulali Aktas, Mehmet Z. Kocak, Haluk Savli \\ Abant Izzet Baysal University Hospital, Department of Internal Medicine, Bolu, Turkey.
}

\begin{abstract}
Aim: Type 2 Diabetes Mellitus (T2DM) is one of the most important chronic conditions in the world that cause great economic burden on healthcare providers. Authors study novel inflammatory markers that derived from routine blood count tests in inflammatory conditions, such as T2DM. One of these parameters is platelet distribution width (PDW), which refers the size variability of circulating platelets. In present retrospective study, we aimed to compare PDW levels of T2DM patients to those with healthy subjects. We also aimed to compare PDW levels of diabetic subjects with and without chronic diabetic complications.
\end{abstract}

Methods: Type 2 diabetic patients admitted to outpatient clinics between January and June 2018 were enrolled to the study.

Results: The PDW of patients with T2DM $(18.1 \pm 0.9 \%)$ was significantly increased compared to the PDW of healthy control subjects (16.7 $\pm 0.5 \%$ ). In subgroup analyze of patients with T2DM revealed that PDW and HbA1c levels of the patients with diabetic nephropathy were significantly elevated compared to the diabetic subjects without diabetic nephropathy ( $p<0.001$ for both HbA1c and PDW). Similarly, PDW and $\mathrm{HbAlc}$ levels of the patients with diabetic neuropathy were significantly elevated compared to the diabetic subjects without diabetic neuropathy $(\mathrm{p}<0.001$ for PDW and $\mathrm{p}=0.01$ for HbA1c). PDW was positively and significantly correlated with $\mathrm{HbA1c}(\mathrm{r}=0.63, \mathrm{p}<0.001)$ and fasting plasma glucose $(\mathrm{r}=0.48, \mathrm{p}<0.001)$ levels.

Conclusion: Elevated PDW should alert physicians for possible T2DM in otherwise healthy subjects. It may be useful in follow up of diabetic regulation and development of diabetic neuropathy and nephropathy in subjects with T2DM.

Keywords: Type 2 diabetes mellitus, Platelet distribution width, HbA1c, Diabetic chronic complications, Hemogram, Fasting plasma glucose, Inflammation.

\section{INTRODUCTION}

Type 2 Diabetes Mellitus (T2DM) is one of the most important chronic conditions in the world that cause great economic burden on healthcare providers [1]. Micro-vascular complications of T2DM, diabetic retinopathy, neuropathy and nephropathy, are responsible from a serious amount of diabetes related morbidity and mortality in this population.

Development and progressions of both diabetic retinopathy, neuropathy and nephropathy are associated with diabetic regulation. Glycated hemoglobin (HbAlc) is widely and universally accepted as a marker of diabetic control in subjects with T2DM. Since the lifespan of the erythrocytes is about 120 days, HbAlc fails to predict acute deteriorations in diabetic control level.

Authors study novel inflammatory markers that derived from routine blood count tests in inflammatory conditions, such as T2DM. Mean platelet volume (MPV), an indice of thrombocyte activation, has been studied in T2DM and found to be elevated in diabetic subjects compared to healthy population $[2,3]$. Besides MPV, MPV to platelet ratio was also linked to the diabetic regulation [4]. Another of these

*Address correspondence to this author at the Abant Izzet Baysal University Hospital, Department of Internal Medicine, Bolu, Turkey.

E-mail: burcinatak@hotmail.com hemogram parameters is platelet distribution width (PDW), which refers the size variability of circulating platelets [5]. Since PDW is not affected by swelling of the thrombocytes, it was considered as a better predictor of platelet activation than MPV [6]. Platelet anisocytosis term is used when the PDW elevated to normal range of 9-14\% [6]. It is increased during platelet activation [7]. PDW has been found to be increased in coronary heart disease and related with the extent of the arterial disease [8].

In present retrospective study, we aimed to compare PDW levels of T2DM patients to those with healthy subjects. We also aimed to compare PDW levels of diabetic subjects with and without chronic diabetic complications.

\section{METHODS}

Type 2 diabetic patients admitted to outpatient clinics between January and June 2018 were enrolled to the study. Patients with active inflammatory conditions; such as rheumatoid arthritis, inflammatory bowel disease, and patients with cancer were excluded. Other exclusion criteria were age younger than 18 years and pregnancy. Control group was consisted of volunteers whom asked a routine check-up on admission to internal medicine outpatient clinics of our institution.

www.njhsciences.com 
General characteristics and physical examination findings of the patients; age, sex, height, body weight, waist circumference, systolic and diastolic blood pressures were recorded. Body mass index (BMI) was calculated with division of body weight by the square of height $(\mathrm{kg} / \mathrm{m} 2)$.

Hemogram parameters; white blood cell count (WBC), hemoglobin $(\mathrm{Hb})$, hematocrit $(\mathrm{Htc})$, platelet count $(\mathrm{Plt})$ and PDW were obtained from patient files and recorded. Similarly, biochemical parameters, such as, $\mathrm{HbAlc}$, fasting blood glucose, serum creatinine, triglyceride, total cholesterol, LDL cholesterol and HDL cholesterol levels were obtained from computerized database and patient files of our institution.

Diabetic microvascular complications for each diabetic participant were researched in patients' files by searching renal functions and proteinuria, ophthalmology consultation and neurology consultation.

All data were analyzed by SPSS software (SPSS 15.0 for Windows, IBM Co. Chicago, IL, USA). Kolmogorov-Smirnov test used to determine distribution of the variables in study groups homogenously or not. Homogenous variables were conducted with $t$ test and expressed as mean \pm standard deviation. Non-homogenously distributed variables were conducted with Mann Whitney U test and expressed as median (min-max). Categorical variables were compared in study cohort by chi-square test. Correlations between study variables were analyzed with Pearson's correlation test. The $p$ values lower than 0.05 are considered as significant.

\section{RESULTS}

Study cohort was consisted of 120 participants, 75 patients with T2DM and 45 healthy controls. Median ages of T2DM and control groups were 53 (29-66) and 52 (30-59) years, respectively $(p=0.12) .39$ of 75 in T2DM group and 31 of 45 in control group were women $(\mathrm{p}=0.07)$.

Diastolic blood pressure $(\mathrm{p}=0.20)$, Plt $(\mathrm{p}=0.62)$, serum creatinine $(\mathrm{p}=0.06), \mathrm{Hb}(\mathrm{p}=0.58), \mathrm{Ht}(\mathrm{p}=0.23)$, and height $(\mathrm{p}=0.10)$ were not significantly different from controls in T2DM group. Table 1 shows the characteristics of the study population.

Table 1. Prevalence of Physical and Non-Physical Violence among Nurses.

\begin{tabular}{|c|c|c|c|c|}
\hline & & T2DM group & Control Group & $\mathbf{p}$ \\
\hline Gender & $\begin{array}{c}\text { Men (n) } \\
\text { Women (n) }\end{array}$ & $\begin{array}{l}36 \\
39\end{array}$ & $\begin{array}{l}14 \\
31\end{array}$ & 0.07 \\
\hline \multicolumn{5}{|c|}{ Median (Min-Max) } \\
\hline $\begin{array}{c}\text { Age (years) } \\
\text { Systolic blood pressure }(\mathrm{mmHg}) \\
\text { Diastolic blood pressure }(\mathrm{mmHg})\end{array}$ & $\begin{array}{c}53(29-66) \\
120(90-180) \\
70(50-110)\end{array}$ & & $\begin{array}{c}52(30-59) \\
120(90-130) \\
70(50-80)\end{array}$ & $\begin{array}{l}0.12 \\
0.02 \\
0.20\end{array}$ \\
\hline \multicolumn{5}{|c|}{ Mean \pm SD } \\
\hline $\begin{array}{c}\text { Body weight }(\mathrm{kg}) \\
\text { Body mass index }(\mathrm{kg} / \mathrm{m} 2) \\
\text { Waist circumference }(\mathrm{cm})\end{array}$ & $\begin{array}{c}82 \pm 12 \\
30,9 \pm 5,5 \\
105 \pm 12\end{array}$ & & $\begin{array}{c}70 \pm 9 \\
25,6 \pm 4,2 \\
88 \pm 12\end{array}$ & $\begin{array}{l}<0.001 \\
<0.001 \\
<0.001\end{array}$ \\
\hline
\end{tabular}

Both weight $(\mathrm{p}<0.001)$, BMI $(\mathrm{p}<0.001)$, waist circumference $(p<0.001)$, systolic blood pressure $(p=0.02)$, WBC $(p<0.001)$, fasting plasma glucose $(p<0.001)$, serum total cholesterol $(p=0.003)$, LDL cholesterol $(p=0.03)$, and triglyceride $(\mathrm{p}<0.001)$ levels were significantly higher in diabetic subjects compared to controls. Serum HDL cholesterol was significantly decreased in T2DM group compared to control group $(\mathrm{p}<0.001)$.

The PDW of patients with T2DM $(18.1 \pm 0.9 \%)$ was significantly increased compared to the PDW of healthy control subjects $(16.7 \pm 0.5 \%)$. The difference between study groups was statistically significant $(\mathrm{p}<0.001)$.

In Pearson's correlation test, PDW was positively and significantly correlated with $\mathrm{HbA1c}(\mathrm{r}=0.63, \mathrm{p}<0.001)$ and fasting plasma glucose $(r=0.48, p<0.001)$ levels (Fig. 1).
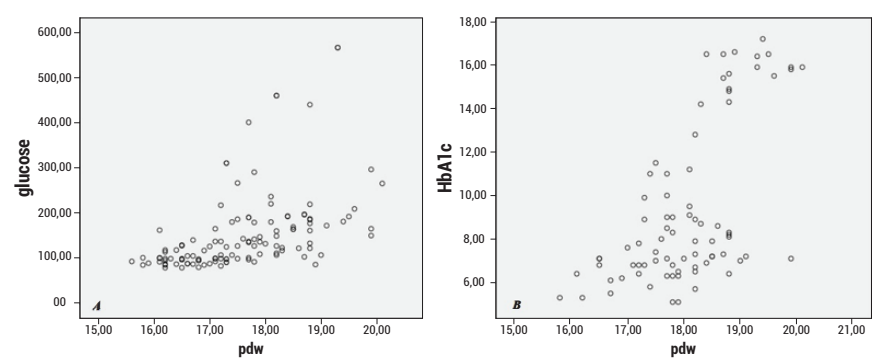

Fig. (1). Correlations between PDW and Fasting Plasma Glucose and PDW and $\mathrm{HbAlc}$.

In subgroup analyze of patients with T2DM revealed that PDW and HbAlc levels of the patients with diabetic 
nephropathy were significantly elevated compared to the diabetic subjects without diabetic nephropathy $(\mathrm{p}<0.001$ for both HbAlc and PDW). Similarly, PDW and HbAlc levels of the patients with diabetic neuropathy were significantly elevated compared to the diabetic subjects without diabetic neuropathy $(\mathrm{p}<0.001$ for PDW and $\mathrm{p}=0.01$ for $\mathrm{HbA1c}$ ). However, PDW and HbAlc levels of the diabetic subjects with and without diabetic retinopathy were not statistically different ( $\mathrm{p}=0.38$ for PDW, $\mathrm{p}=0.24$ for HbA1c).

\section{DISCUSSION}

We showed that PDW was significantly higher in type 2 diabetic patients compare to healthy population. Another striking result of present study is that, similar to the HbAlc, PDW was increased in T2DM subjects with diabetic neuropathy and diabetic nephropathy compared to diabetics without these chronic complications.

Elevated PDW values have been reported to be related with increased mortality in patients with acute myocardial infarction that treated with primary percutaneous coronary intervention and authors suggested PDW over $16 \%$ as independent prognostic factor for this population [6].

There are several reports about elevation in PDW in immune thrombocytopenia, an autoimmune condition [9].

Studies on cardiac conditions have revealed that PDW was higher in patients with ST segment elevation myocardial infarction compared to the subjects with stable coronary artery disease [10]. Moreover, increased PDW was suggested as an independent predictor of thrombolysis failure in patients with ST segment elevation myocardial infarction [10].

Authors from India reported that PDW was significantly increased in subjects with coronary disease compared to the subjects with normal angiographic findings [11]. On the other hand, greater PDW values were detected in patients with stable coronary heart disease that have total coronary occlusion compared to the subjects without total occlusion of coronary artery [12]. Another study concluded that Ihara et al. reported that PDW was significantly elevated in coronary disease with positive angiography results compared to those with negative angiographic findings [13].

Besides coronary disease, elevated PDW has been studied in diabetes mellitus, too. It has been reported in 2011 that, PDW was elevated in patients with diabetes mellitus compared to control group [14]. Moreover, they found that PDW was greater in diabetics with microvascular complications than the diabetics without any microvascular complications [14]. However, they did not compared PDW in diabetics with diabetic complications to those without separately as diabetic retinopathy, neuropathy and nephropathy. Our study differ from theirs being compared each microvascular complications in subjects with and without them. In addition they did not analyzed the correlation between $\mathrm{HbAlc}$ and PDW, which have been strongly correlated to each other in our present study. HbA1c reflects diabetic regulation in 3 months period since the lifespan of erythrocytes is about 120 days. Since the life of platelets is 7-10 days, PDW may reflect change in diabetic regulation with shorter time periods than HbA1c.

We have showed that PDW was significantly higher in T2DM patients with diabetic nephropathy or neuropathy than the PDW in diabetics without these complications. We could not showed such a significant PDW elevation in T2DM subjects with diabetic retinopathy compared to those without which we think was due to the small number of diabetics with diabetic retinopathy (only seven patients had diabetic retinopathy).

Although we showed elevated PDW in T2DM, there are some contrast studies in literature, too. Beyan et al. suggested that PDW was not correlated with platelet aggregation [15]. However, many other reports in literature suggest our findings $[16,17]$. In a recent study by Jabeen et al, authors concluded that increased levels of PDW could be helpful in indicating poor glycemic control in diabetic population [18]. Moreover, subjects with diabetic chronic complications had higher PDW values compared to the diabetics without chronic complications [19].

Elevated PDW in diabetic subjects may indicate other adverse events. $\mathrm{Hu}$ et al. reported that increased PDW was an independent predictor of stent restenosis in diabetic coronary artery disease subjects [20].

Two important limitations of our work are relatively small study population and retrospective design of the study. However, we believe that the results of present study, elevated PDW in T2DM with diabetic neuropathy or diabetic nephropathy, are very important for current literature.

\section{CONCLUSION}

In conclusion, elevated PDW should alert physicians for possible T2DM in otherwise healthy subjects. It may be useful in follow up of diabetic regulation and development of diabetic neuropathy and nephropathy in subjects with T2DM.

\section{CONFLICT OF INTEREST}

Declared none.

\section{ACKNOWLEDGEMENTS}

Declared none.

\section{REFERENCES}

[1] Chen L, Magliano DJ, Zimmet PZ. The worldwide epidemiology of type 2 diabetes mellitus-present and future perspectives. Nat Rev Endocrinol 2012; 8(4): 228.

DOI: 10.1038 /nrendo.2011.183 
[2] Aktas G, Kocak MZ, Duman TT, et al. Mean platelet volume (MPV) as an inflammatory marker in type 2 diabetes mellitus and obesity. Bali Med J 2018; 7(3): 650-3.

[3] Demirtunc R, Duman D, Basar M, Bilgi M, Teomete M, Garip $\mathrm{T}$. The relationship between glycemic control and platelet activity in type 2 diabetes mellitus. J Diabetes Compl 2009; 23(2): 89-94. DOI: 10.1016/j.jdiacomp.2008.01.006

[4] Duman TT, Aktas G, Atak B, Kocak MZ. Is mean platelet volume to platelet ratio a promising indicator of diabetic regulation in type 2 diabetes mellitus? J Med Res 2018; 4(3): 137-9.

[5] Osselaer J-C, Jamart J, Scheiff J-M. Platelet distribution width for differential diagnosis of thrombocytosis. Clin Chem 1997; 43: 1072-16.

[6] Rechcinski T, Jasinska A, Forys J, et al. Prognostic value of platelet indices after acute myocardial infarction treated with primary percutaneous coronary intervention. Cardiol J 2013; 20(5): 491-8. DOI: 10.5603/CJ.2013.0134

[7] Vagdatli E, Gounari E, Lazaridou E, Katsibourlia E, Tsikopoulou F, Labrianou I. Platelet distribution width: A simple, practical and specific marker of activation of coagulation. Hippokratia 2010; 14(1): 28-32.

[8] De Luca G, Venegoni L, Iorio S, et al. Platelet distribution width and the extent of coronary artery disease: Results from a large prospective study. Platelets 2010; 21(7): 508-14. DOI: $10.3109 / 09537104.2010 .494743$

[9] Gardner FH, Bessman JD. Thrombocytopenia due to defective platelet production. Clin Haematol 1983; 12(1): 23-38.

[10] Cetin M, Bakirci EM, Baysal E, et al. Increased platelet distribution width is associated with ST-segment elevation myocardial infarction and thrombolysis failure. Angiology 2014; 65(8): 737-43. DOI: 10.1177/0003319713520068

[11] Khandekar MM, Khurana AS, Deshmukh SD, Kakrani AL, Katdare AD, Inamdar AK. Platelet volume indices in patients with coronary artery disease and acute myocardial infarction: An Indian scenario. J Clin Pathol 2006; 59(2): 146-9. DOI: $10.1136 /$ jcp.2004.025387

[12] Vatankulu MA, Sonmez O, Ertas G, et al. A new parameter predicting chronic total occlusion of coronary arteries: Platelet distribution width. Angiology 2014; 65(1): 60-4.

DOI: $10.1177 / 0003319713486339$

[13] Ihara A, Kawamoto T, Matsumoto K, Shouno S, Morimoto T, Noma Y. Relationship between hemostatic factors and the platelet index in patients with ischemic heart disease. Pathophysiol Haemostas Thrombos 2006; 35(5): 388-91.

DOI: $10.1159 / 000097694$

[14] Jindal S, Gupta S, Gupta R, et al. Platelet indices in diabetes mellitus: indicators of diabetic microvascular complications. Hematology (Amsterdam, Netherlands) 2011; 16(2): 86-9. DOI: $10.1179 / 102453311 X 12902908412110$

[15] Beyan C, Kaptan K, Ifran A. Platelet count, mean platelet volume, platelet distribution width, and plateletcrit do not correlate with optical platelet aggegation responses in healthy volunteers. J Thrombos Thrombol 2006; 22(3): 161-4.

DOI: $10.1007 / \mathrm{s} 11239-006-9014-7$

[16] Buch A, Kaur S, Nair R, Jain A. Platelet volume indices as predictive biomarkers for diabetic complications in Type 2 diabetic patients. J Lab Phys 2017; 9(2): 84-8.

DOI: $10.4103 / 0974-2727.199625$

[17] Zaccardi F, Rocca B, Pitocco D, Tanese L, Rizzi A, Ghirlanda G. Platelet mean volume, distribution width, and count in type 2 diabetes, impaired fasting glucose, and metabolic syndrome: A meta-analysis. Diabetes Metab Res Rev 2015; 31(4): 402-10. DOI: $10.1002 / \mathrm{dmrr} .2625$

[18] Jabeen F, Fawwad A, Rizvi HA, Alvi F. Role of platelet indices, glycemic control and hs-CRP in pathogenesis of vascular complications in type-2 diabetic patients. Pak J Med Sci 2013; 29(1): 152-6.

[19] Shilpi K, Potekar RM. A study of platelet indices in type 2 diabetes mellitus patients. Indian J Hematol Blood Transfus 2018; 34(1): 115-20. DOI: 10.1007/s12288-017-0825-9

[20] Hu CP, Du Y, Zhu Y, Shi C, Qin Z, Zhao YX. Platelet Distribution width on admission predicts in-stent restenosis in patients with coronary artery disease and type 2 diabetes mellitus treated with percutaneous coronary intervention. Chin Med J 2018;131:757-63. DOI: 10.4103/0366-6999.228247

Received: June 28, 2018 